\title{
ACCIÓN DE MICROORGANISMOS EFICIENTES SOBRE LA ACTIVIDAD DE INTERCAMBIO CATIÓNICO EN PLÁNTULAS DE ACACIA (ACACIA MELANOXYLOM) PARA LA RECUPERACIÓN DE UN SUELO DEL MUNICIPIO DE MONDOÑEDO, CUNDINAMARCA
}

\author{
Effect of efficient microorganisms on cation exchange capacity in acacia \\ seedlings (Acacia melanoxylon) for soil recovery in Mondoñedo, Cundinamarca
}

Palabras clave: capacidad de intercambio catiónico, bases intercambiables, microorganismos eficientes., compost, mulch, gallinaza, fertilización química.

Keywords: cation exchange capacity, alcali exchange, efficient microorganisms, compost, mulch, gallinaza, chemical fertilization.

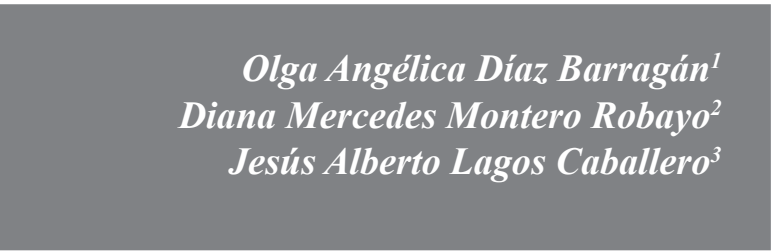

\section{RESUMEN}

Se determinó la acción microorganismos eficientes (EM) sobre la capacidad de intercambio catiónico (CIC) para la recuperación de un suelo del municipio de Mondoñedo, Cundinamarca. Se instaló una unidad de invernadero para mantener condiciones estables. Luego de la recolección, el tamizado y la homogenización de la muestra de suelo, se realizaron análisis físicos y químicos iníciales. Para el montaje de unidades experimentales se obtuvieron plántulas de Acacia melanoxylon provenientes de Zabrinsky. Se realizó un diseño completamente aleatorizado con ocho tratamientos y tres repeticiones. Para el mantenimiento y seguimiento del comportamiento de las plantas se halló una frecuencia de riego de tres veces por semana. La aplicación del EM se hizo durante tres meses: el primer mes, se aplicó cuatro veces (una vez por semana), el segundo mes, se aplicó dos veces (una vez cada quince días) y el tercer mes se hizo una sola aplicación. Adicionalmente se hicieron análisis morfológicos cada 15 días, (registro de número de hojas, ramas y diámetro del tallo). Al final se tomaron muestras del suelo de cada matera y se llevaron al laboratorio para hacer los análisis correspondientes de CIC, bases intercambiables, saturación de bases, relaciones entre elementos y tejido vegetal, haciendo lecturas de los elementos en el espectrofotómetro de absorción atómica.
Se hizo un análisis estadístico de pruebas de comparaciones múltiples y de varianza que mostraron diferencias significativas entre los tratamientos. De esta forma se encontró la mejor alternativa que pretende mejorar la calidad ambiental de los suelos erosionados como los del desierto de Zabrinsky: fue EM en dosis de 5\% del agua del riego, mejorando el incremento de la CIC en el suelo, con la mezcla de los abonos orgánicos (compost, mulch y gallinaza) en dosis de una libra cada uno, y con la fertilización química, compuesta por manganeso electrolítico $(0.0002 \mathrm{~g})$, cobre $(0.0002 \mathrm{~g})$, zinc (0.0001g), URFOS 44 (166.66g) y klip-boro(5g).

\begin{abstract}
We determined the effect of efficient microorganisms (EM) on the cation exchange capacity for soil recovery in the municipality of Mondoñedo, Cundinamarca. A greenhouse unit was installed in order to maintain stable conditions. After harvesting, sifted and homogenization of the soil sample, initial physical and chemical analyses were made. For the experimental units we used Acacia melanoxylon seedlings from Zabrinsky. A completely randomized design was done with eight treatments and three repetitions. For the maintenance and monitoring of the seedlings behaviour, a frequency of irrigation of three times per week was found. The
\end{abstract}

1 Universidad de La Salle. kikadiazb@hotmail.com Universidad de La Salle. dianamonterorobayo@yahoo.com

Universidad Distrital Francisco José De Caldas. Correspondencia: jalagosc@udistrital.edu.co jeallaca@yahoo.com 
application of the EM was done during three months: in the first month, it was applied four times (once a week); during the second month, it was applied twice (biweekly), and during the third month there was only one application. Additionally, every 15 days morphological analyses were made (number of leaves, branches and stem diameter).

In the end, soil samples were taken from each plant pot. In the laboratory we analysed the cation exchange capacity, alkali ion exchange, saturation alkali, relations between elements and plant tissue. These were done using an atomic absorption spectrophotometer.

Statistical analyses consisted on multiple comparisons test and variance tests, in order to find whether or not treatments exhibited significant differences. In that way, the best alternative for improving environmental quality of eroded soils as the Zabrinsky desert is the efficient microorganisms in 5\% doses in irrigation water. Additionally, the cation exchange capacity must be enhanced using organic fertilizers (compost, mulch and gallinaza) in one pound doses, and chemical fertilizers: electrolytic $\mathrm{Mn}$ (0.0002 g), Cu (0.0002 g), Zn (0.0001 g), URFOS $44(166.66 \mathrm{~g})$ and klip-boro $(5 \mathrm{~g})$.

\section{INTRODUCCIÓN}

Esta es una investigación que estudia la recuperación de los suelos degradados, específicamente en el desierto Zabrinsky, ubicado en la localidad de Mondoñedo, municipio de Mosquera, Cundinamarca, con el biofertilizante EM (microorganismos eficientes), que, según estudios, permite mejorar las propiedades físicas, químicas y biológicas de todo tipo de suelos (Higa 1994).

Por lo tanto, se aplicó el biofertilizante EM a plántulas de Acacia melanoxylon con el fin de evaluar específicamente su acción sobre la capacidad de intercambio catiónico y conocer qué otras prácticas combinadas con este compuesto podrían utilizarse para la aceleración de la recuperación del suelo objeto de estudio. Se dispusieron ocho tratamientos involucrando otros abonos orgánicos como el compost, el mulch, la gallinaza y fertilización química mezclados con EM, para finalmente determinar la mejor opción y dar solución al problema planteado.

\section{METODOLOGÍA}

El proceso de investigación se llevó a cabo en la Universidad de la Salle, sede centro, en el área denominada "Los Pinos", donde se instaló una unidad de invernadero con el objetivo de mantener condiciones ambientales estables.

El suelo fue recolectado en la localidad del desierto de Zabrinsky, ubicado en el municipio de Mosquera, Cundinamarca. Se recolectó un volumen de muestra de $48 \mathrm{~kg}$ aproximadamente del epipedón (horizonte A) y $48 \mathrm{~kg}$ aproximadamente del endopedón (horizonte B). Se determinó que el suelo pertenece al orden taxonómico Alfisol, el cual tiene un epipedón mólico sobre un endopedón argílico. El nombre taxonómico asignado al suelo en estudio es Typic Haplustalfs; esta clasificación fue realizada por los autores siguiendo las claves de la Keys to Soil Taxonomy (2006).

Con el fin de obtener una muestra homogénea, con características granulométricas similares, y para garantizar uniformidad en el diseño experimental, la muestra de suelo (horizontes A y B, respectivamente) fue tamizada con tamiz de $2 \mathrm{~mm}$ de diámetro.

El análisis físico de textura del suelo se realizó en el laboratorio de suelos de la Universidad Nacional de Colombia (metodología de Bouyoucus); los análisis de densidad real y de densidad aparente se hicieron en el laboratorio de Ingeniería Ambiental de la Universidad de la Salle (técnica de terrón en parafina y picnómetro, respectivamente), al igual que los análisis químicos de capacidad de intercambio catiónico (CIC), saturación de bases y pH. La capacidad de intercambio catiónico y la determinación de bases cambiables se realizaron mediante la metodología del acetato de amonio. Para la determinación de bases cambiables se utilizó el espectrofotómetro de absorción atómica del laboratorio de Ingeniería Ambiental de la Universidad de la Salle. Tanto las variables físicas como quími- 
cas se determinaron dos veces durante el desarrollo de la investigación, siguiendo las metodologías del Instituto Geográfico Agustín Codazzi (IGAC 1990). La primera a los dos meses o 60 días (mitad del proceso investigativo de invernadero) y la otra una vez finalizado o a los 120 días (cuatro meses). De las materas se tomó únicamente el primer horizonte para realizar los respectivos análisis.

Se definieron los siguientes tratamientos, cada uno con tres repeticiones: T1, testigo (suelo sin tratamiento); T2, EM; T3, compost con EM; T4, mulch con EM; T5, gallinaza con EM; T6, fertilización química con EM; T7, compost con mulch, gallinaza y EM, y T8, compost con mulch, gallinaza, fertilización química y EM. En cuanto a la dosis de EM, y por sugerencia de los fabricantes del producto Fundases (2006), se determinó que lo más adecuado era el siguiente procedimiento: el primer mes, cada ocho días al 5\%; el segundo mes, cada 15 días al $5 \%$, y al tercer mes una sola dosis al 5\%. La cantidad de abono orgánico usado fue de una libra y los elementos para el tratamiento de fertilización química se adicionaron según las necesidades planteadas por Chapman (1980).

El resultado de los análisis del suelo inicial (Tabla 1), realizados por la Universidad Nacional de Colombia, se compararon con los niveles sugeridos por Chapman (1980). Con lo anterior se determinaron las deficiencias de los elementos que se tuvieron en cuenta para el tratamiento de fertilización química, los cuales fueron: nitrógeno $(\mathrm{N}): 30 \mathrm{~g}$; fósforo $(\mathrm{P}): 30 \mathrm{~g}$; cobre $(\mathrm{Cu}): 0.002 \mathrm{~g}$; manganeso (Mn): 0.002 g; Zinc (Zn): 0.001; Boro (B): 5 g.

Para calcular la cantidad de agua a aplicar, y teniendo en cuenta la fuerza de retención de agua en las plantas, se realizó una prueba de riego de la siguiente forma: se pusieron dos materas con suelo en una bandeja y a cada una de las materas se les aplicó $2000 \mathrm{ml}$ de agua destilada para llevar la relación 1:1 (2 kg de suelo en cada matera: $2000 \mathrm{ml}$ de agua) y se dejó allí 24 horas.

Tabla 1. Análisis de suelo inicial.

\begin{tabular}{|c|c|c|c|c|c|c|c|c|c|}
\hline \multirow{2}{*}{ pH } & \multirow{2}{*}{$\mathrm{Ca} \mathrm{cmol} / \mathrm{k}$} & \multirow{2}{*}{$\mathrm{K} \mathrm{cmol} / \mathrm{k}$} & \multirow{2}{*}{$\mathrm{Mg} \mathrm{cmol} / \mathrm{k}$} & \multirow{2}{*}{$\mathrm{Na} \mathrm{cmol} / \mathrm{k}$} & \multicolumn{3}{|c|}{ Testura FArA* } & \multirow{2}{*}{ MO \% } & \multirow{2}{*}{$\mathrm{CIC}$} \\
\hline & & & & & $\operatorname{Ar} \%$ & $\mathbf{L} \%$ & A $\%$ & & \\
\hline 6.09 & 8.05 & 0.83 & 4.07 & 0.41 & 30 & 22 & 49 & 7.05 & 24.1 \\
\hline
\end{tabular}

*Franco arcilloso arenoso

Como resultado de esta prueba en la bandeja escurrieron $1400 \mathrm{ml}$ de agua, lo que determinó que la absorción del suelo fue de $600 \mathrm{ml}$. Esta cantidad se aplicó a cada planta con una frecuencia de riego de ocho días. Se obtuvieron plántulas de característica similar (20 cm de altura aproximadamente) de Acacia melanoxylon provenientes del desierto de Zabrinsky para plantarlas en las materas.

Una vez homogenizado el suelo (cada horizonte por aparte), se hizo el montaje en las materas, poniendo $1 \mathrm{~kg}$ del horizonte B y sobre éste $1 \mathrm{~kg}$ de suelo del horizonte A. Se aclara que se homogenizó el suelo de cada matera con el tratamiento especifico (excluyendo el mulch, porque éste debió ir en forma de capa encima del horizonte A). Una vez se tuvo la matera con el tratamiento incorporado, se trasplantaron las plantas de Acacia melanoxylon.

La investigación se dividió en dos partes: a los 60 días se reemplazaron las plántulas, con el objetivo de realizar un primer análisis químico de tejido. A estas primeras plantas se les denominó "antiguas". Luego se resembró otro grupo de plántulas, en las mismas materas, que duraron otros dos meses. A éstas se les denominó "plantas nuevas". De igual forma, a este segundo grupo se le realizó un análisis químico de tejido vegetal.

En cuanto a las características morfológicas, se tuvieron en cuenta el diámetro del tallo, número de ramas y número de hojas. Además se llevó un registro de temperatura ambiental dentro de la uni- 
dad experimental. El estudio morfológico se realizó cada 15 días. Cuando se retiraban las plántulas (a los 2 y 4 meses) se tomó el dato de peso de la planta con raíz y sin ella (este peso se denomina "peso húmedo"). Una vez obtenido el dato del peso húmedo, las plantas se guardaron para tomar el peso seco (se secaron a $60^{\circ} \mathrm{C}$ durante 24 horas) y hacer el análisis de tejido vegetal respectivo.

Se acordó emplear un diseño experimental completamente aleatorizado (DCA) con ocho tratamientos y tres repeticiones. Para los análisis estadísticos de suelo y tejido vegetal de plantas se realizó un análisis multivariado de varianza (MANOVA), en el cual las variables respuesta $(\mathrm{Yj})$ correspondían a la medición de calcio $(\mathrm{Ca})$, magnesio $(\mathrm{Mg})$, sodio $(\mathrm{Na})$, potasio $(\mathrm{K})$, manganeso $(\mathrm{Mn})$, hierro $(\mathrm{Fe})$ y cobre $(\mathrm{Cu})$ en las plantas, en meq/100g, y la variable explicativa (Ti), al tipo de tratamiento aplicado al suelo. De igual forma, las variables de respuesta (Yj) en el suelo correspondían a las bases, sus relaciones, la cIc y el pH. Y la variable explicativa (Ti), al tipo de tratamiento aplicado al suelo. Para su análisis, estos valores se compararon con la tabla de referencia dada por la Universidad de California (Chapman1980) y del Instituto Geográfico Agustín Codazzi (Igac, 1990).

Modelo MAnOva:

$\mathrm{Yj}=\mathrm{Ti} \operatorname{con} \mathrm{j}=1 \ldots, \mathrm{n}$ e $\mathrm{i}=1 \ldots 8$ donde: $\mathrm{Yi}=\left[\mathrm{Y}_{1}\right.$, $\left.\mathrm{Y}_{2}, \mathrm{Yn}\right]$ y $\mathrm{Tj}=\left[\mathrm{T}_{1}, \mathrm{~T}_{2}, \mathrm{~T}_{3}, \mathrm{~T}_{4}, \mathrm{~T}_{5}, \mathrm{~T}_{6}, \mathrm{~T}_{7}, \mathrm{~T}_{8}\right]$.

La hipótesis nula planteada es:

$\mathrm{H} 0=\mathrm{T}_{1}=\mathrm{T}_{2}=\mathrm{T}_{3}=\mathrm{T}_{4}=\mathrm{T}_{5}=\mathrm{T}_{6}=\mathrm{T}_{7}=\mathrm{T}_{8}$.

Lo que significa que los tratamientos son iguales entre sí o, en otras palabras, que no existen diferencias estadísticamente significativas entre ellos. A su vez, la hipótesis alternativa sostiene que al menos un tratamiento es diferente de los demás: $\mathrm{H}_{1}=$ al menos un Ti es diferente de los demás con $\mathrm{i}=1 \ldots, 8$.

Para los elementos (variables de respuesta) en que la hipótesis nula del análisis de varianza fue rechazada con evidencia estadística, los resultados se sometieron a pruebas de comparación múltiples entre tratamientos de Duncan para identificar el (los) tratamiento(s) que difería(n) de los demás. El nivel de significancia de la prueba estadística se manejó como F significativa a $\mathrm{P}=0.05$ y $\mathrm{P}=0.01$ (ver resultados generales del análisis de varianza y prueba de Duncan en anexos correspondientes). Para el procesamiento de los datos se utilizó el programa estadístico SAs (Statistical Analysis System).

\section{RESULTADOS Y DISCUSIÓN}

\section{MUESTRA DE SUELO INICIAL}

En la Tabla 1 se puede observar que el análisis del suelo arrojó un pH de 5.7, valor que está clasificado como suelo medianamente ácido. Hay alta disponibilidad de calcio $(\mathrm{Ca})$, potasio $(\mathrm{K})$ y magnesio $(\mathrm{Mg})$, capacidad de intercambio catiónico (CIC) y fósforo $(\mathrm{P})$. Se encontró nivel medio para $\mathrm{N}$ y carbono orgánico (CO), y nivel óptimo para sodio. Con respecto a los microelementos se encontró que en el nivel crítico estaba zinc, sobre el nivel crítico hierro y por debajo de él cobre y manganeso. La textura a la que pertenece el suelo de Zabrinsky es FArA (franco arcilloso arenoso).

\section{CAPACIDAD DE INTERCAMBIO CATIÓNICO}

El análisis de CIC a la mitad del proceso se realizó transcurridos dos meses de haber comenzado la aplicación de EM . En la Figura 1a se observa que la muestra inicial es superior a la del tratamiento1 (testigo), mientras que el valor inicial con respecto al resto de los tratamientos es menor.

En general, la CIC aumenta porque la aplicación de fertilización química (compuesta por manganeso electrolítico, boro, cobre, zinc, URFOS 44, klip-boro) aportó unos niveles importantes de bases. Esto se corrobora observando que el tratamiento con mayor CIC es el T8 (abonos orgánicos + EM + fertilización química) y que en segundo lugar se encuentra el T6 (fertilización química). En tercer lugar se encuentra el T3 (gallinaza + EM), el cual presenta la mayor cantidad de bases. En cuarto lugar se encuentra el compost, que también aporta bases. En quinto lugar debería estar el mulch, pero es el EM el que sobrepasa este último elemento, debido a que probablemente el mulch no ha llevado a cabo su 
proceso de mineralización. La disminución del T7 (abonos orgánicos + EM) es una prueba más de que la fertilización química es la que está aportando las bases. Esto contradice lo que afirma Higa (1994) acerca de que el EM no funciona en presencia de fertilizantes químicos.
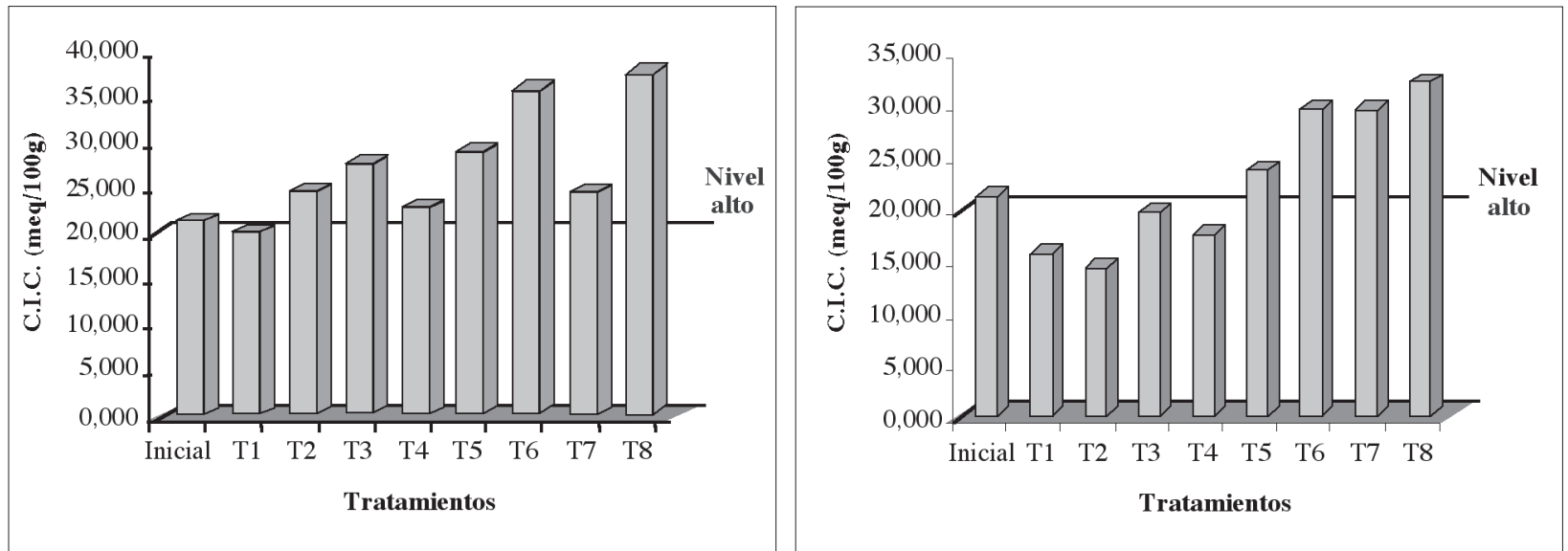

Figura 1. a. Capacidad de intercambio catiónico obtenida a los 60 días. b. Capacidad de intercambio catiónico obtenida a los 120 días. T1 (testigo), T2 (EM), T3 (compost + EM), T4 (mulch + EM), T5 (gallinaza), T6 (fertilización química), T7 (abonos orgánicos + EM), T8 (abonos orgánicos + EM + fertilización química).

Se detectaron diferencias estadísticas altamente significativas $(1 \%)$ de $\mathrm{p}=0.0030$ entre los tratamientos. Por lo tanto se rechaza la hipótesis nula (Anexo A). Para ratificar esta afirmación se corrieron las pruebas de comparación de Duncan y se encontró que los tratamientos T6 (fertilización química) y T8 (abonos orgánicos + EM + fertilización química) tienen una marcada diferencia en comparación con el testigo.

La Figura $1 \mathrm{~b}$ muestra una disminución de la CIC en los tratamientos T1 (testigo), T2 (EM), T3 (Compost + EM) y T4 (mulch + EM) con respecto a la muestra inicial, debido a que a los cuatro meses de iniciar el proceso las plantas ya han absorbido los cationes de cambio ( $\mathrm{Ca}, \mathrm{Mg}, \mathrm{K}, \mathrm{Na}$ ) que se encontraban retenidos en la materia orgánica. Los demás tratamientos tienen valores superiores al de la muestra inicial.

Comparando con los resultados obtenidos a los dos meses de iniciado el proceso, se observa que aunque los tratamientos conservan el mismo comportamiento la CIC disminuyó en general, atribu- yéndose esto a que las plantas han absorbido las bases. No obstante, el T7 (abonos orgánicos + EM) presenta un aumento, debido a que el EM en presencia de los abonos orgánicos (compost, mulch, gallinaza) hace que se aumente la CIc. El valor de probabilidad realizado por el MANOVA para CIC fue altamente significativo (1\%) de 0.0001 , indicando que estadísticamente sí hay diferencias entre tratamientos, aceptando la hipótesis alternativa. Esto se corroboró con lo encontrado al correr la prueba de comparación de Duncan (Anexo B).

pH

El análisis de varianza para la variable $\mathrm{pH}$ mostró una probabilidad de 0.0001 , lo que indicó claramente diferencias significativas entre tratamientos, aceptando la hipótesis alternativa. Esto se verificó con las pruebas de comparaciones realizadas. Duncan encontró diferencias en los T4 (mulch + EM), T5 (gallinaza), T6 (fertilización química), T7 (abonos orgánicos + EM) y T 8 (abonos orgánicos + EM + fertilización química) en comparación con el testigo. 

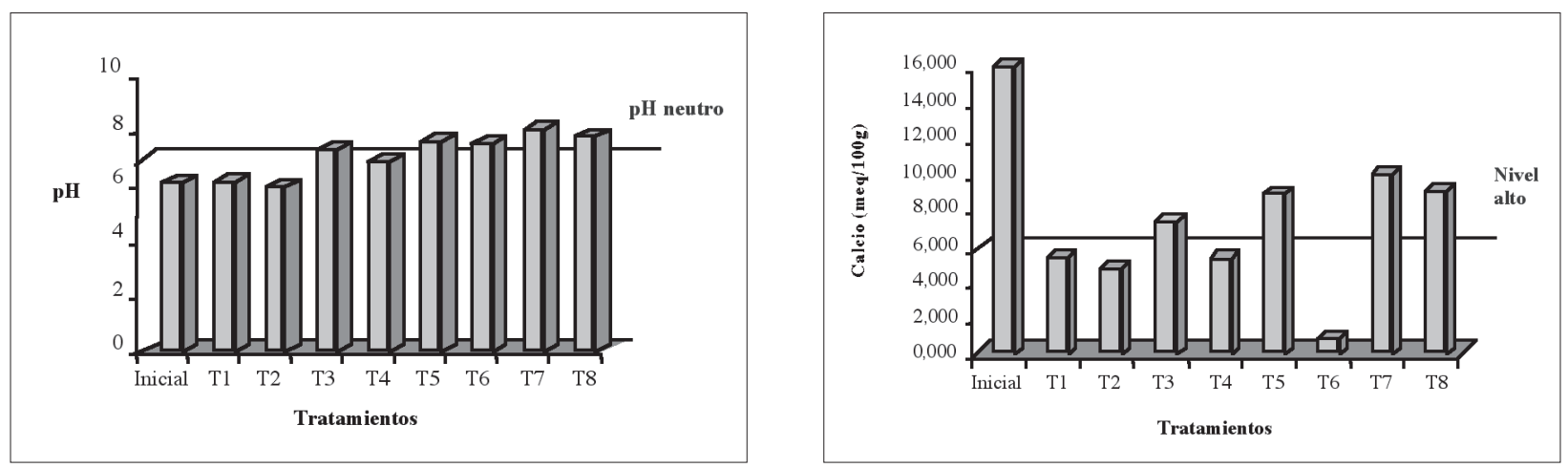

Figura 2. a. Resultados de $\mathrm{pH}$ en el suelo por tratamiento a los 120 días. b. Variación de calcio (meq/100g) por tratamiento en el suelo a los 120 días. T1 (testigo), T2 (EM), T3 (compost + EM), T4 (mulch + EM), T5 (gallinaza), T6 (fertilización química), T7 (abonos orgánicos + EM), T8 (abonos orgánicos + EM + fertilización química).

$\mathrm{Al}$ observar la Figura 2a, se comprueba que el pH pasó de ser moderadamente ácido a ligeramente alcalino en la muestra inicial y en los tratamientos T2 (EM), T5 (gallinaza + EM), T6 (fertilización química + EM), T7 (abonos orgánicos + EM) y T8 (abonos orgánicos $+\mathrm{EM}+$ fertilización química). Los tratamientos T3 (compost + EM) y T4 (mulch + EM) se encontraron con $\mathrm{pH}$ neutro. El valor de $\mathrm{pH}$ de los tratamientos T5, T7 y T8 está relacionado con el alto contenido de calcio que tiene la gallinaza, ya que estos tratamientos reportaron altos niveles en el análisis foliar.

En comparación con la Figura 1a de CIC a la mitad del proceso (60 días), se observa que existe una relación entre los tratamientos que aumentaron $\mathrm{pH}$ con los tratamientos que aumentaron su capacidad de intercambio catiónico. Lo anterior es explicado por Marín (1990): "La carga negativa del humus aumenta a reacciones más alcalinas. La carga negativa del humus aumenta linealmente con el $\mathrm{pH}$, siendo cerca de cero a $\mathrm{pH}$ de 3 a 4 y de 100 a pH 6. Se estima que la CIC de la materia orgánica a $\mathrm{pH}$ 8.2 , es de alrededor de $200 \mathrm{meq} / 100 \mathrm{~g}$ ". Además se observa en la Figura 2a cómo el EM tiende a disminuir el pH (comportamiento de acidez residual) cuando es aplicado solo.

Por otra parte, el fertilizante químico tiende a generar una acidez residual (bajo $\mathrm{pH}$ ) o una basicidad residual ( $\mathrm{pH}$ básico) según el tipo de fertilizante que se aplique (ICA 1992). Para este caso, los pro- ductos químicos aplicados en combinación con el EM generaron basicidad residual.

\section{BASES INTERCAMBIABLES}

El análisis de varianza muestra una probabilidad de 0.0001, lo cual indicó que hay grandes diferencias entre tratamientos, rechazando la hipótesis nula. Al aplicar las pruebas de comparación de Duncan entre tratamientos, se presentaron cuatro grupos, confirmando así el comportamiento de las variables antes analizadas.

El calcio disminuyó notablemente para todos los tratamientos con relación a la muestra inicial, debido a que este nutriente posiblemente fue absorbido por la planta como se observa en la Figura $2 b$

Según el ICA (1992), el T6 está en un rango bajo $(0.892 \mathrm{meq} / 100 \mathrm{~g})$, porque en este tratamiento se aplicó fertilización química (compuesta por Mn, B, $\mathrm{Cu}, \mathrm{Zn}, \mathrm{P}$ y nitrógeno) con EM, además de que este elemento tiene un comportamiento antagonista con los microelementos aplicados, como es el caso del zinc (Graetz 1996). Los tratamientos T1, T2 y T4 están en un nivel medio que corresponden a testigo, aplicación de EM y mulch + EM, respectivamente.

Los demás tratamientos se encuentran en un nivel alto: el tratamiento T3 (compost + EM), el T5 (gallinaza + EM), que aporta gran cantidad de calcio, y los tratamientos T7 (compost + mulch + gallinaza 
+ EM) y T8 (compost + mulch + gallinaza + fertilizante químico + EM) también favorecen el aumento de esta variable, por ser la combinación de los demás tratamientos, mejorando las condiciones físico-químicas del suelo (Proexant 2005).

\section{Calcio (Ca) en relación Suelo - Planta}
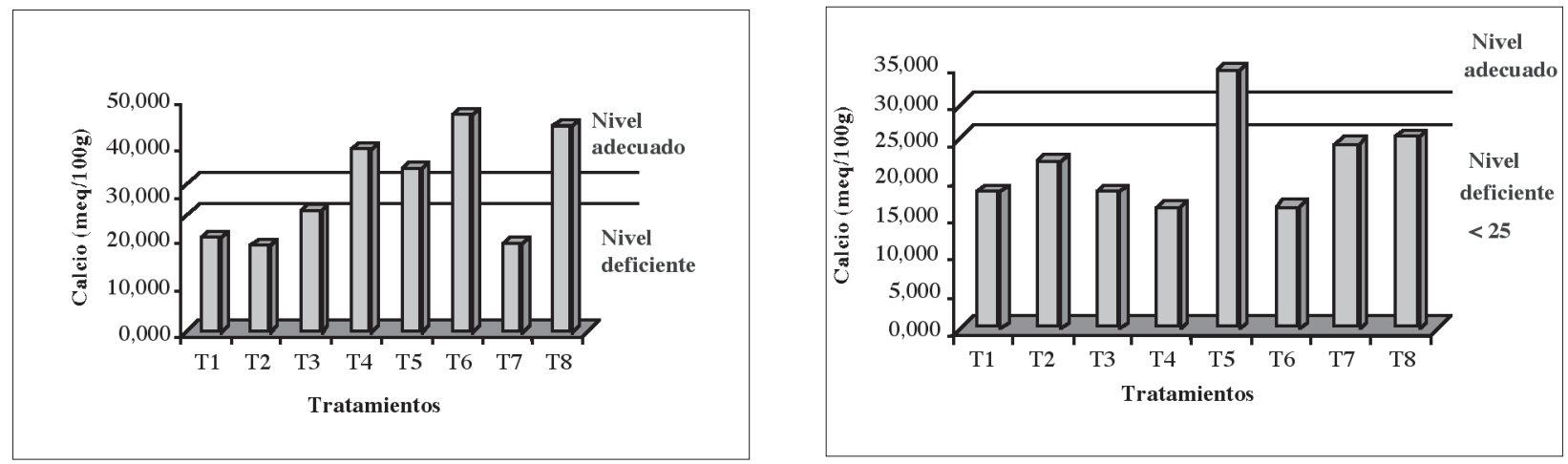

Figura 3. a. Calcio en tejido de plantas antiguas. b. Calcio en tejido de plantas nuevas. T1 (testigo), T2 (EM), T3 (compost + EM), T4 (mulch + EM), T5 (gallinaza), T6 (fertilización química), T7 (abonos orgánicos + EM), T8 (abonos orgánicos + EM + fertilización química).

Al finalizar la investigación se presentó una relación inversamente proporcional, ya que las plantas nuevas absorbieron más calcio que las plantas antiguas, como se muestra en las Figuras 3a (variación del calcio en plantas antiguas) y $3 \mathrm{~b}$ (variación del calcio en plantas nuevas), exceptuando en los tratamientos T2 (EM) y T7 (compost + mulch + gallinaza $+\mathrm{EM})$, donde las plantas antiguas absorbieron más que las nuevas, y en el T5 (gallinaza + EM), donde plantas antiguas y nuevas mantuvieron el mismo nivel de absorción debido a la aplicación de gallinaza + EM.

Las plantas antiguas de todos los tratamientos, menos el T5 (gallinaza + EM), y las nuevas de los tratamientos T1 (testigo), T2 y T7 tienen niveles deficientes de calcio según Chapman (1980). De acuerdo con este autor, las plantas nuevas de los tratamientos T4, T5, T6 y T8 tienen niveles adecuados de esta base.

De lo anterior se deduce que EM en plantas antiguas no contribuye a que haya una absorción apropiada de Ca cuando se aplica solo, con abonos orgánicos como mulch y compost, en mezcla con otros abonos orgánicos (compost + mulch + gallinaza) o en mezcla de abonos orgánicos + fertilización química. Se obtienen niveles apropiados de calcio en plantas antiguas al aplicar EM con gallinaza. Esto se puede explicar a partir del análisis de abonos aplicados, donde se observa que la gallinaza tiene un valor de $428.750 \mathrm{meq} / 100 \mathrm{~g}$ de calcio.

Tabla 2. Análisis de los abonos aplicados.

\begin{tabular}{|c|c|c|c|c|c|c|c|}
\hline Abono & $\begin{array}{c}\text { Calcio } \\
\text { meq/100g }\end{array}$ & $\begin{array}{l}\text { Magnesio } \\
\mathrm{meq} / 100 \mathrm{~g}\end{array}$ & $\begin{array}{c}\text { Sodio } \\
\text { meq/100g }\end{array}$ & $\begin{array}{c}\text { Potasio } \\
\text { meq/100g }\end{array}$ & $\begin{array}{c}\text { Hierro } \\
\text { meq/100g }\end{array}$ & $\begin{array}{c}\text { Manganeso } \\
\mathrm{meq} / 100 \mathrm{~g}\end{array}$ & $\begin{array}{c}\text { Cobre } \\
\text { meq/100g }\end{array}$ \\
\hline EM en dilución & 2.238 & 0.018 & 0.4870 & 2.603 & 0.037 & 0.003 & 0.001 \\
\hline Compost & 150.550 & 1.239 & 2.7087 & 64.447 & 17.893 & 2.439 & 0.043 \\
\hline Mulch & 32.950 & 0.271 & 1.1261 & 7.979 & 0.611 & 0.075 & 0.026 \\
\hline \multirow[t]{2}{*}{ Gallinaza } & 428.750 & 3.529 & 5.8022 & 87.847 & 1.131 & 1.735 & 0.091 \\
\hline & 614.488 & 5.058 & 10.124 & 162.877 & 19.672 & 4.252 & 0.161 \\
\hline
\end{tabular}


Como se puede constatar con el análisis morfológico (ver Tablas 3 y 4), se observa cómo el perímetro, tanto en plantas nuevas como antiguas, disminuye ratificando lo de Calderón (2000), donde una deficiencia de calcio puede inhibir el desarrollo de los tallos de las plantas.

Tabla 3. Perímetro ( $\mathrm{mm})$ en plantas antiguas: T1 (testigo), $\mathrm{T} 2$ (EM), T3 (compost + EM ), T4 (mulch + EM), T5 (gallinaza), T6 (fertilización química), T7 (abonos orgánicos + EM), T8 (abonos orgánicos + EM + fertilización química).

\begin{tabular}{ccc}
\hline Tratamiento & $\begin{array}{c}\text { Primera lectura } \\
(\mathbf{m m})\end{array}$ & $\begin{array}{c}\text { Lectura a los } \\
\text { dos meses }(\mathbf{m m})\end{array}$ \\
\hline T1 & 33.20 & 19.63 \\
T2 & 31.88 & 17.33 \\
T3 & 29.07 & 11.50 \\
T4 & 22.58 & 11.67 \\
T5 & 16.73 & 11.00 \\
T6 & 27.29 & 14.00 \\
T7 & 31.37 & 16.00 \\
T8 & 26.03 & 15.33 \\
\hline
\end{tabular}

Tabla 4. Perímetro (mm) en plantas nuevas: T1 (testigo), T2 (EM), T3 (compost + EM), T4 (mulch + EM), T5 (gallinaza), T6 (fertilización química), T7 (abonos orgánicos + EM), T8 (abonos orgánicos + EM + fertilización química).

\begin{tabular}{ccc}
\hline Tratamiento & $\begin{array}{c}\text { Primera lectura } \\
(\mathbf{m m})\end{array}$ & $\begin{array}{c}\text { Lectura a los dos meses } \\
(\mathbf{m m})\end{array}$ \\
\hline \hline T1 & 23.12 & 15.00 \\
T2 & 20.38 & 12.50 \\
T3 & 18.95 & 11.67 \\
T4 & 16.61 & 13.33 \\
T5 & 17.93 & 7.00 \\
T6 & 20.23 & 5.67 \\
T7 & 16.17 & 8.00 \\
T8 & 16.25 & 8.67 \\
\hline
\end{tabular}

El tratamiento T6, que presenta menor valor de $\mathrm{Ca}$ en el suelo (Figura 2b), concuerda con la planta nueva (Figura $3 b$ ), que tiene más Ca en su tejido foliar y, en general, las plantas nuevas que se encuentran en suelos que contienen fertilizante químico + EM absorbieron más calcio; por lo tanto, se dedujo que la fertilización química es la que contribuye a que estas plantas absorban más calcio.

Ni las plantas nuevas ni las antiguas presentan niveles tóxicos de calcio a nivel foliar, ya que nin- guna alcanza un valor cercano a $87.5 \mathrm{meq} / 100 \mathrm{~g}$. Esto se ratifica por los niveles dados por Chapman (1980). El análisis estadístico determina una probabilidad de 0.0239 , que indica la existencia de diferencias entre tratamientos pero, al no ser ese valor contundente, se aplicó la prueba de comparación de Duncan.

Como resultado de esta prueba, se confirma que efectivamente hay diferencias significativas entre tratamientos, determinándose la hipótesis alternativa para esta variable. Estadísticamente se considera que hay diferencias significativas entre tratamientos, porque el análisis arrojó un valor de 0.0985 para F, es decir que se acepta la hipótesis alternativa. Esta afirmación se confirmó claramente mediante la prueba de comparación de Duncan.

\section{Magnesio (Mg)}

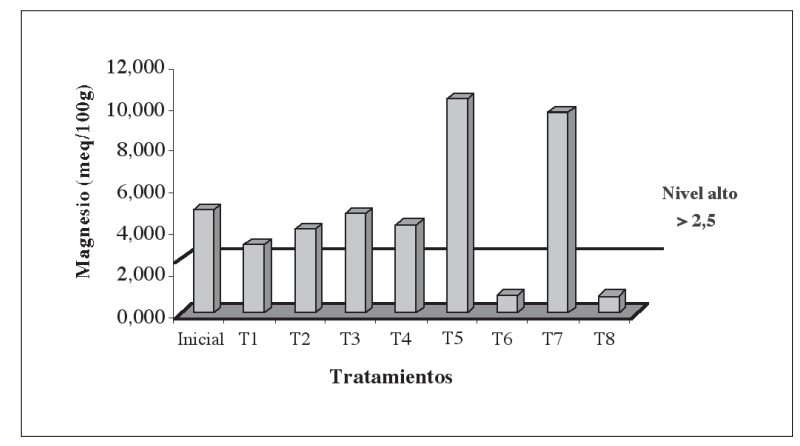

Figura 4. Variación de magnesio por tratamiento: T1 (testigo), T2 (EM), T3 (compost + EM), T4 (mulch + EM), T5 (gallinaza), T6 (fertilización química), T7 (abonos orgánicos + EM), T8 (abonos orgánicos + EM + fertilización química).

La Figura 4 indica que la muestra inicial y los tratamientos T1 (testigo), T2 (EM), T3 (Compost + EM), T4 (Mulch + EM), T5 (gallinaza + EM) y T7 (abonos orgánicos $+\mathrm{EM})$ se encuentran dentro de un rango alto, y los tratamientos T6 (fertilización química + EM) y T8 (abonos orgánicos + fertilización química + EM) están en un nivel bajo. Lo anterior se debe a que los fertilizantes químicos no proporcionan ni permiten la fijación del magnesio en el suelo (Guerrero 1995).

El análisis de varianza arrojó un valor de probabilidad de 0.0001 , que evidencia diferencias significativas entre tratamientos, aceptando la hipótesis alternativa. Posteriormente se aplicaron las prue- 
bas de comparación de medias de Duncan, las cuales reflejaron que efectivamente las diferencias significativas están en los T6 y T8, que obtuvieron valores de 0.8025 y 0.7915 respectivamente.

\section{Magnesio (Mg) en relación suelo - planta}
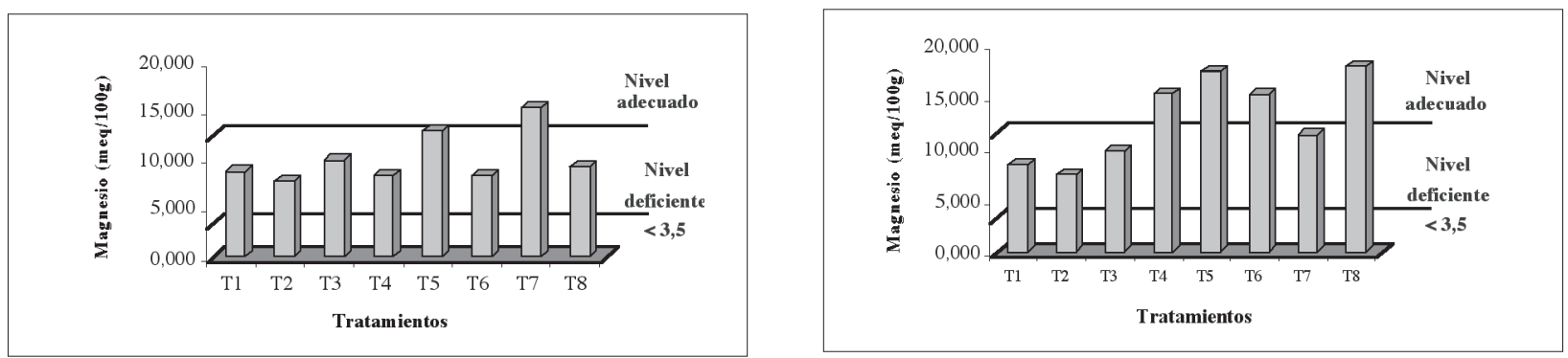

Figura 5. a. Magnesio en tejido en plantas antiguas. b. Magnesio en tejido en plantas nuevas. T1 (testigo), T2 (EM), T3 (compost + EM), T4 (mulch + EM), T5 (gallinaza), T6 (fertilización química), T7 (abonos orgánicos + EM), T8 (abonos orgánicos $+\mathrm{EM}+$ fertilización química).

Lo observado en las Figuras 5a y 5b se explica en que la gallinaza aporta mayor contenido de magnesio que los otros abonos orgánicos en plantas antiguas y nuevas. EM aplicado con compost no facilita el incremento de magnesio para las plantas porque a los cuatro meses de investigación no se ha dado el proceso de mineralización. Igualmente, EM aplicado solo no funciona. El mejor tratamiento es el $\mathrm{T} 7$ en plantas antiguas, atribuido esto a la mezcla de los abonos orgánicos con EM, y en plantas nuevas es el T8, debido tal vez a su fisionomía.

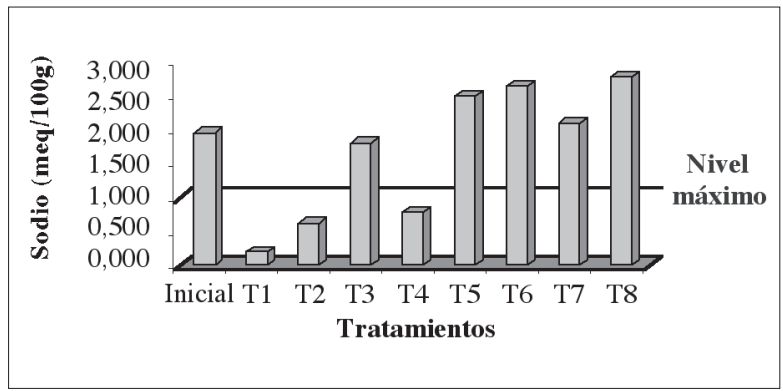

Figura 6. Variación de sodio por tratamiento. T1 (testigo), T2 (EM.), T3 (compost + EM), T4 (mulch + EM), T5 (gallinaza), T6 (fertilización química), T7 (abonos orgánicos + EM), T8 (abonos orgánicos + EM + fertilización química).

El análisis de la variable magnesio para plantas antiguas muestra un valor $\mathrm{F}$ de 0.1429 , que indi- ca la existencia estadística de algunas diferencias entre tratamientos. Para comprobar esto se aplicó el análisis de comparación entre variables de Duncan, el cual dio como resultado que, aunque no son marcadas, sí existen diferencias entre tratamientos. Entonces se acepta la hipótesis alternativa.

\section{Sodio (Na)}

Un contenido apropiado de sodio en el suelo debe ser menor a valores de 1.0 (ICA 1992). Estas cantidades encontradas hacen que el suelo se torne tóxico, ocasionando que la planta no absorba los iones de igual forma. Se encuentran en un valor óptimo los tratamientos T1 (testigo), T2 (EM.) y T4 (mulch $+\mathrm{EM})$.

La variable sodio muestra una probabilidad de 0.0104 , lo que demuestra que hay diferencias significativas entre los tratamientos, reconociendo entonces la existencia de una hipótesis alternativa. La prueba de comparación de medias de Duncan también permitió encontrar que los tratamientos T3 (compost + EM), T5 (gallinaza + EM), T6 (fertilización química + EM), T7 (abonos orgánicos + EM) y T8 (abonos orgánicos + fertilización química + EM) están fuera del límite, mientras que los tratamientos T1 (testigo), T2 (EM), y T4 (mulch + EM) están dentro del rango permisible. 


\section{Sodio (Na) en relación suelo - planta}

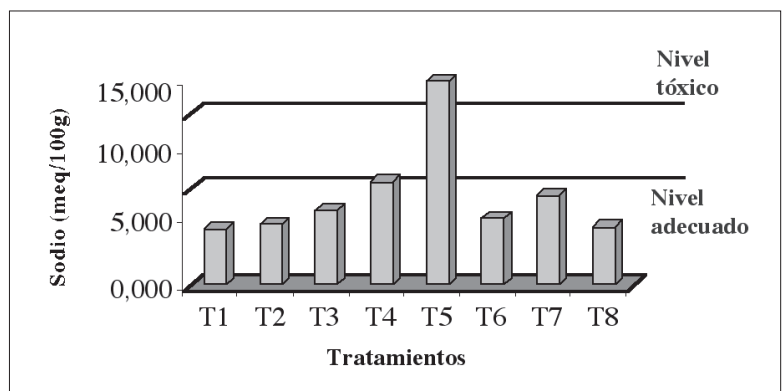

Figura 7. a. Sodio en tejido en plantas antiguas. b. Sodio en tejido en plantasnuevas. T1 (testigo), T2 (EM), T3 (compost + EM), T4 $($ mulch + EM), T5 (gallinaza), T6 (fertilización química), T7 (abonos orgánicos + EM), T8 (abonos orgánicos + EM + fertilización química).

Como se observó en la Figura 6, los tratamientos T5 (gallinaza + EM), T6 (fertilización química + EM) y T8 (abonos orgánicos + fertilización química + EM) son los que presentan mayor contenido de sodio en el suelo, y por esta razón las plantas que se observaron con niveles tóxicos son aquellas que pertenecen a estos tratamientos.

En la Figura 7a, que corresponde a plantas antiguas, el T5 alcanza un nivel tóxico, atribuido en parte al alto contenido de sodio de la gallinaza, tal como se muestra en el análisis de abonos aplicados (Tabla 8), y a que absorbió sodio del suelo. Los demás tratamientos se encuentran dentro de un nivel adecuado.

El análisis para esta variable es de 0.1099, determinando que estadísticamente no hay diferencias significativas entre todos los tratamientos. Para confirmar esto, se realizó la prueba de comparación de Duncan, que permitió encontrar que esta apreciación es totalmente cierta. Para este caso, se dice que se acepta la hipótesis alternativa.

Con relación a la gráfica de sodio en plantas nuevas (Figura 7b), se observa que los tratamientos T6 (fertilización química + EM) y T8 (abonos orgáni$\mathrm{cos}+\mathrm{EM}+$ fertilización química) presentan niveles tóxicos, porque hay aportes del suelo y de la fertilización química. En tercer lugar se observó el tratamiento que contiene gallinaza + EM. De manera general, todos los tratamientos que presentan valores superiores a 1.0 en el suelo coinciden con la descripción dada para los análisis foliares.

El análisis de varianza señala que para la variable sodio existe una diferencia entre los tratamientos, pero como el valor no era contundente, se aplicaron las pruebas de comparación de Duncan. Estas pruebas encontraron que el valor más alto lo tiene el T8 (combinación de abonos orgánicos + Fertilizantes químicos + EM) con 20.708, mientras que el EM tiene el valor más bajo (2.814).

\section{Potasio (K)}

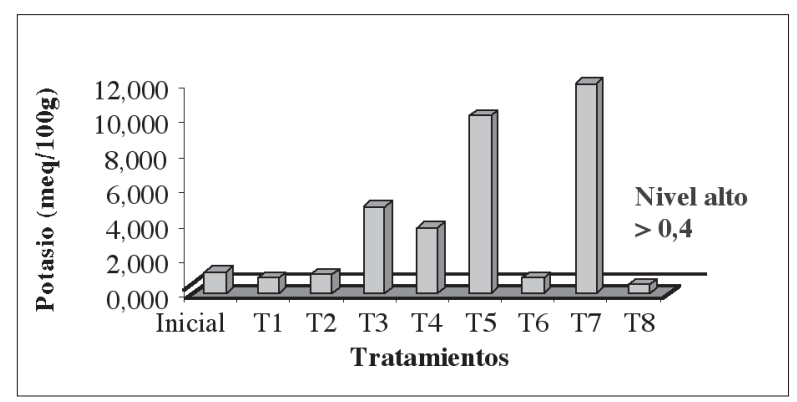

Figura 8. Variación de potasio por tratamiento: T1 (testigo), T2 (EM), T3 (compost + EM), T4 (mulch + EM), T5 (gallinaza), T6 (fertilización química), T7 (abonos orgánicos + EM), T8 (abonos orgánicos + EM + fertilización química).

La Figura 8 indica que todos los tratamientos, incluyendo la muestra inicial, están en un alto rango, según (ICA 1992). Para potasio, según Chapman (1980), se considera que un suelo es tóxico cuando se tiene un valor mayor a $0.6 \mathrm{meq} / 100 \mathrm{~g}$. Por esta razón todos los tratamientos, excepto el T8, se encuentran en esta condición. Sin embargo, se establece que los valores más altos, como los de los tratamientos T3 (compost + EM), T5 (gallinaza + EM) y T7 (abonos orgánicos + EM), están dados de acuerdo a la alta disponibilidad de materia orgánica, pues actúan como activador de carbohidratos (León 2000). Mientras que los demás tratamientos están relacionados con la fertilización química 
o, como en el caso de T1 (testigo) y T2 (EM) que contienen baja concentración de materia orgánica, también se pudo dar que el potasio fue absorbido por las plantas.

Como indica Estrada (2000), la secuencia de retención catiónica es $\mathrm{Ca}++>\mathrm{Mg}++>\mathrm{Na}+>\mathrm{k}+$, $\mathrm{y}$ generalmente es el orden de abundancia de las bases cambiables. Esto explica que en el suelo se absorban más el calcio, el magnesio y el sodio,

\section{Potasio (K) en relación suelo - planta}

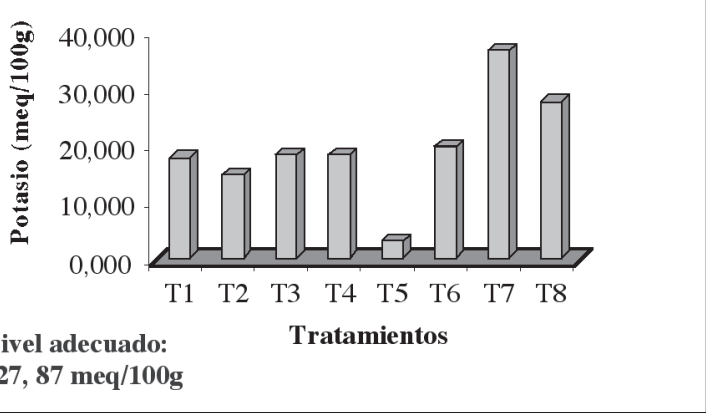

que a su vez pueden ser absorbidos por las plantas, mientras que el potasio no.

El análisis de varianza para potasio muestra una probabilidad de 0.0001 , lo que quiere decir que hay diferencias significativas entre tratamientos. Esto se confirma con los resultados obtenidos en la prueba de comparación de Duncan, la cual indicó que los tratamientos T3 (compost + EM), T5 (gallinaza + EM) y T7 (abonos orgánicos + EM) tienen los valores más altos.

Figura 9. a. Potasio en tejido de plantas antiguas. b. Potasio en tejido de plantas nuevas.T1 (testigo), T2 (EM), T3 (compost + EM), T4 (mulch + EM), T5 (gallinaza), T6 (fertilización química), T7 (abonos orgánicos + EM), T8 (abonos orgánicos + EM + fertilización química).

Todos los tratamientos, tanto en plantas antiguas como nuevas, presentan deficiencia de potasio (menor a $102.3 \mathrm{meq} / 100 \mathrm{~g}$ ). Esto se puede explicar por el efecto antagónico del magnesio sobre el potasio: como explica Lora (1984), puede haber una disminución en el rendimiento de la planta debido al exceso de magnesio. La Figura 9a explica que el tratamiento que presentó mayor nivel de absorción de potasio en las plantas antiguas fue el T7 (abonos orgánicos $+\mathrm{EM})$, mientras que el de menor absorción fue el T5 (gallinaza + EM).

El valor obtenido del análisis estadístico para esta variable es de 0.0133 , lo que determina que no existen diferencias significativas entre tratamientos. Esta apreciación se contradice al realizar la prueba de comparación de Duncan, la cual dio como re- sultado que hay grandes diferencias entre todos los tratamientos, y ratificó una hipótesis alternativa.

El análisis de la variable potasio en plantas nuevas muestra que el T8 (abonos orgánicos $+\mathrm{EM}+$ fertilización química) presentó mayor absorción y, en general, en las plantas nuevas se encontró mayor cantidad de potasio que en las antiguas, debido a su fisionomía y a que su aspecto radical era mejor en el momento de la siembra (Figura 9b).

El análisis de varianza muestra una probabilidad para el valor $F$ de 0.2957 , lo que significa que estadísticamente hay diferencias entre tratamientos. Por esta razón se rechaza la hipótesis nula. Esto se confirma con la prueba de Duncan que no arrojó comparaciones importantes. 


\section{SATURACIÓN DE BASES}

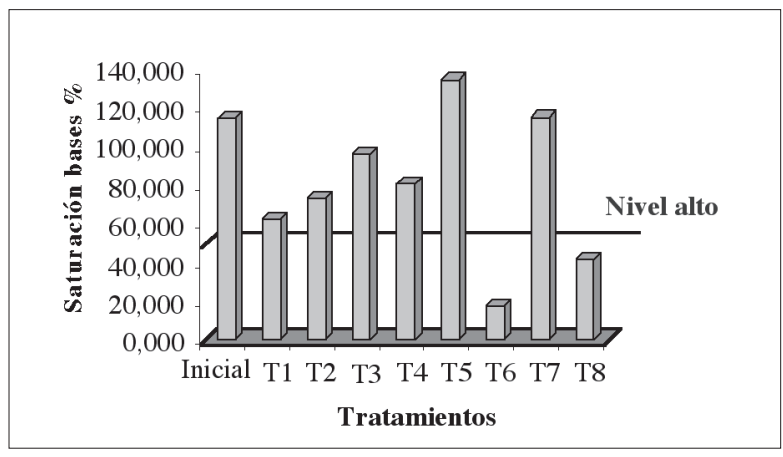

Figura 10. Porcentaje de saturación de bases: T1 (testigo), T2 ( EM), T3 (compost + EM), T4 (mulch + EM), T5 (gallinaza), T6 (fertilización química), T7 (abonos orgánicos + EM), T8 (abonos orgánicos + EM + fertilización química).

Según el IGAC (1990) el tratamiento T6 se encuentra en un nivel bajo de saturación de bases, el T8 en nivel medio y el resto de los tratamientos en nivel alto (Figura 10). Este análisis confirma que la proporción de cationes cambiables constituido por bases aumenta a medida que el suelo se saliniza. Comparando la gráfica de saturación de bases (Figura 10) y la de sodio (Figura 6) en el suelo, se presenta un comportamiento inversamente proporcional, exceptuando los tratamientos T6 y T8, lo que quiere decir que la fertilización química baja los niveles de algunas bases en el suelo y los au- menta en las plantas. Cuando el porcentaje de saturación de bases está por debajo del $35 \%$, como en el T6, se dice que hay poca disponibilidad de nutrientes para las plantas. En análisis de varianza la probabilidad fue de 0.0001 , lo que indica que hay diferencias significativas entre tratamientos y demuestra la existencia de una hipótesis alternativa. La confirmación de esta apreciación se da porque la prueba del rango múltiple de Duncan muestra que efectivamente hay grandes diferencias entre los tratamientos.

\section{ANÁLISIS DE RELACIONES ENTRE ELEMENTOS}

Para la interpretación de los resultados de la relación $\mathrm{Ca} / \mathrm{Mg}$ se toma como base la interpretación dada por el IGAC (1990), según el cual la relación $\mathrm{Ca} / \mathrm{Mg}$ en estudio se encuentra por debajo del nivel ideal para todos los tratamientos, es decir, por debajo de 2, con excepción del T8 (abonos orgánicos + fertilizantes químicos $+\mathrm{EM})$, que tiene un valor elevado, correspondiente a 11.40 , que fomentala mayor absorción de calcio (Figura 11a).

Para el T5 (gallinaza + EM) se observa un valor de 0.857 . Esto quiere decir que se presenta un exceso de magnesio para este tratamiento; según Calderón (2000), este exceso provoca reducción en el crecimiento de la planta y distribución irregular de clorofila.

\section{$\mathrm{Ca} / \mathrm{Mg}$ y $\mathrm{Ca} / \mathrm{K}$}
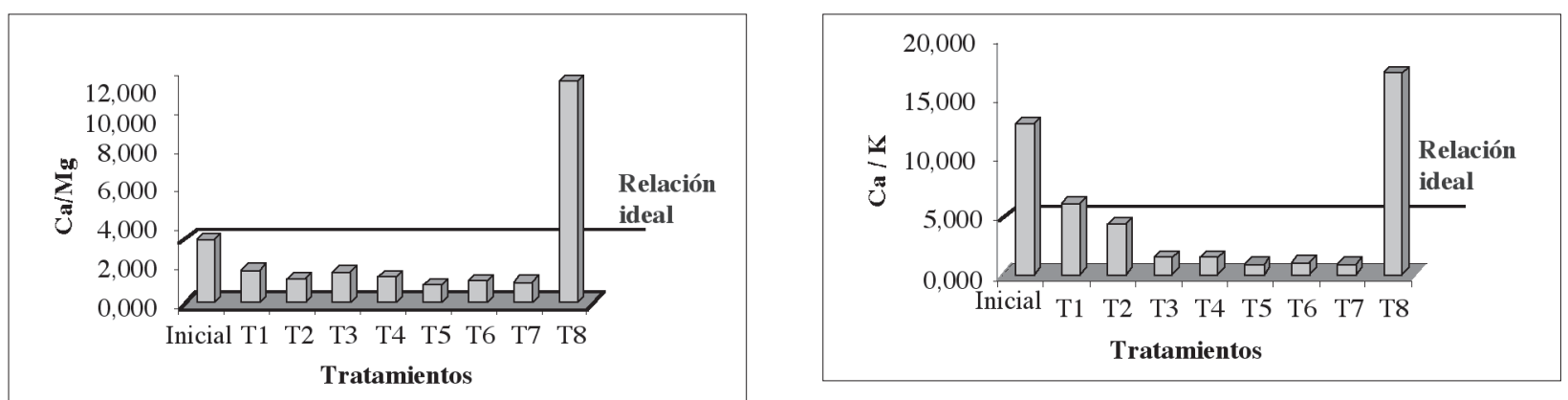

Figura 11. a. Relación $\mathrm{Ca} / \mathrm{Mg}$ entre tratamientos. b. Relación $\mathrm{Ca} / \mathrm{K}$ entre tratamientos. T1 (testigo), T2 (EM), T3 (compost + EM), T4 (mulch + EM), T5 (gallinaza), T6 (fertilización química), T7 (abonos orgánicos + EM), T8 (abonos orgánicos + $\mathrm{EM}+$ fertilización química).

En general, cuando la relación $\mathrm{Ca} / \mathrm{Mg}$ es invertida (valores inferiores a 3) puede provocar una absorción alta de magnesio, y en tal caso pueden presentarse síntomas de toxicidad de $\mathrm{Mg}$, en lugar de deficiencias 
de calcio (Marín 1990). La relación $\mathrm{Ca} / \mathrm{Mg}$ ideal se presenta entonces únicamente para la muestra de suelo inicial, que tiene un valor de 3.244.

Analizando el efecto de la relación $\mathrm{Ca} / \mathrm{Mg}$ con los valores de $\mathrm{pH}$ se tiene que los tratamientos T6, T7 y T8, donde la absorción de calcio es mayor que la de magnesio, coinciden con lo mencionado por Marín (1990) acerca de que en suelos con $\mathrm{pH}$ superiores a 7.3 la absorción de calcio es dependiente de la relación $\mathrm{Ca} / \mathrm{Mg}$ y que cuando se presenta un $\mathrm{pH}$ inferior a 7.3 la absorción de calcio y magnesio no son mayormente afectadas por la relación $\mathrm{Ca} / \mathrm{Mg}$.

Otro aspecto directamente afectado por la relación $\mathrm{Ca} / \mathrm{Mg}$ es la producción primaria bruta (PPB), que está determinada por el aumento en el número de ramas, hojas y grosor del tallo, aspectos importantes a tener en cuenta en la reforestación de un suelo. Dicha producción aumenta cuando la relación $\mathrm{Ca} / \mathrm{Mg}$ es mayor.

El análisis de varianza y la prueba $\mathrm{F}$ para esta relación muestra una probabilidad de 0.0793 , valor que reflejó diferencias significativas entre los tratamientos y confirmó una hipótesis alternativa; pero para saber cuáles eran los tratamientos que representaban dichas diferencias se hicieron las pruebas de comparación de Duncan y ciertamente se encontró que es el tratamiento T8 (abonos orgánicos + ferti- lizantes químicos + EM) el que contiene una mayor diferencia con respecto a los demás. El suelo inicial y el T8 (abonos orgánicos + EM + fertilización química) presentan valores muy bajos de potasio, promoviendo más al absorción de calcio; mientras que los tratamientos T5 (gallinaza + EM) y T7 (abonos orgánicos + EM) presentan valores bajos de calcio, promoviendo más la absorción de potasio.

Se considera que para que haya deficiencia de potasio la relación $\mathrm{Ca} / \mathrm{Mg}$ debe ser mayor a $30 \mathrm{meq} /$ $100 \mathrm{~g}$. Por lo tanto, en el suelo de estudio, el potasio no es deficiente. La aplicación de EM sólo lleva a una relación de $\mathrm{Ca} / \mathrm{K}$ aproximada a la ideal.

El análisis estadístico para la relación $\mathrm{Ca} / \mathrm{K}$ muestra una probabilidad de 0.0001 , lo que indica que hay diferencias significativas entre tratamientos y confirma lo descrito para la Figura $11 \mathrm{~b}$, rechazando así la hipótesis nula. Las pruebas de comparación también encontraron estas mismas diferencias.

\section{Relación $\mathrm{Mg} / \mathrm{K}$ y $(\mathrm{Ca}+\mathrm{Mg}) / \mathrm{K}$}

En la Figura 12a se observan valores cercanos al óptimo según el IGAC (1990) para la muestra de suelo inicial y para los tratamientos T1 (testigo) y T2 (EM), debido a que carecen de alto contenido de materia orgánica, además de presentar valores de pH menores a los de los demás tratamientos.
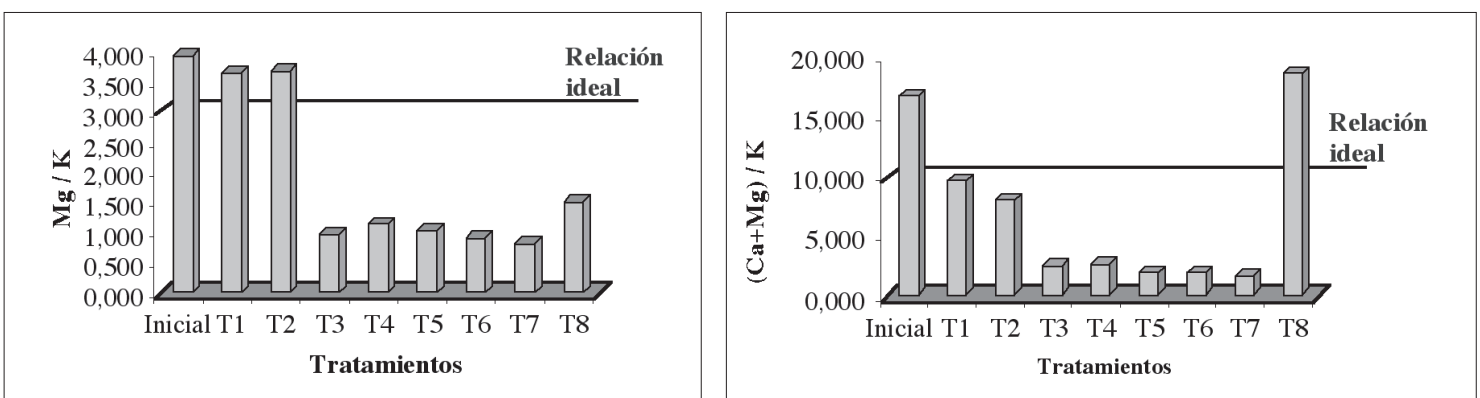

Figura. 12 a. Relación $\mathrm{Mg} / \mathrm{K}$ entre tratamientos. b. Relación $(\mathrm{Ca}+\mathrm{Mg}) / \mathrm{K}$ entretratamientos. T1 (testigo), T2 (EM), T3 $($ compost + EM), T4 (mulch + EM), T5 (gallinaza), T6 (fertilización química), T7 (abonos orgánicos + EM), T8 (abonos orgánicos $+\mathrm{EM}+$ fertilización química).

Los demás tratamientos presentan deficiencias en la relación $\mathrm{Ca} / \mathrm{Mg}$, porque, al contrario de los tratamientos anteriores, contienen bastante mate- ria orgánica, además de tener una alta capacidad de retención de agua que hace que el suelo se compacte. 
Los tratamientos T3 (compost + EM), T6 (fertilización química + EM) y T7 (mezcla de abonos orgánicos + EM) presentan deficiencia de magnesio según la tabla del IGAC. Como se mencionó, este exceso provoca desaceleración en el crecimiento de la planta y distribución irregular de clorofila (Calderón 2000).

La probabilidad encontrada mediante el análisis de varianza es de 0.0001 , cifra que corrobora las diferencias que hay entre tratamientos y la existencia de una hipótesis alternativa. La prueba de comparación de Duncan indica que, en comparación con el testigo, los tratamientos T3 (compost + EM), T4 (mulch + EM), T5 (gallinaza + EMG), T6 (fertilización química), T7 (abonos orgánicos $+\mathrm{EM})$ y T8 (abonos orgánicos + fertilización química $+\mathrm{EM}$ ) presentan diferencias significativas.

Según se observa en la Figura 12b, se presenta una evidente disminución de la relación $(\mathrm{Ca}+\mathrm{Mg}) / \mathrm{K}$ con respecto a la muestra de suelo inicial, excepto el en T8 (abonos orgánicos + fertilización química + EM), debido a que hay alta retención de nutrientes por ser una combinación de materia vegetal, con fertilización química y EM, donde hay mayor absorción de calcio y magnesio que de potasio.

El IGAC (1990) considera 10 un valor óptimo para la relación $(\mathrm{Ca}+\mathrm{Mg}) / \mathrm{K}$. De acuerdo con esta apreciación el valor que más se acerca es el que pertenece al tratamiento 1, es decir, el testigo. El valor que sigue (7.979) es el del T2, correspondiente a aplicación de EM, que mantiene un nivel aproximado al ideal, lo que quiere decir que este tratamiento mantiene unas condiciones favorables para que se presente esta relación.

Para la relación $(\mathrm{Ca}+\mathrm{Mg}) / \mathrm{K}$ la probabilidad de que haya una hipótesis alternativa es muy alta, pues el análisis estadístico muestra un valor $\mathrm{F}$ de 0.0001 , lo que significa que hay diferencias significativas entre tratamientos.

\section{ANALISIS DE TEJIDO VEGETAL DE Fe, Mn y Cu}

\section{Plantas nuevas}
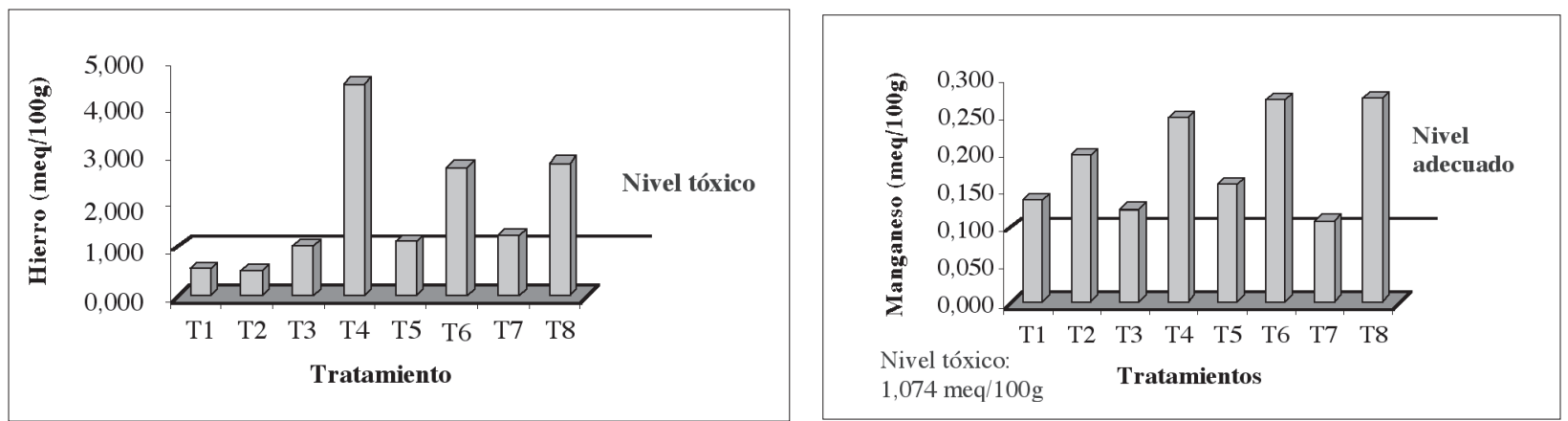

Figura 13. a. Hierro en tejido de plantas nuevas. b. Manganeso en tejido de plantas nuevas. T1 (testigo), T2 (EM), T3 (compost + EM), T4 (mulch + EM), T5 (gallinaza), T6 (fertilización química), T7 (abonos orgánicos + EM), T8 (abonos orgánicos + EM + fertilización química).

Un factor importante que define esta variable es el $\mathrm{pH}$. A mayor $\mathrm{pH}$, menos disponibilidad de hierro. Esta afirmación se puede corroborar observando la Figura 2a. El hierro gana solubilidad cuando hay más humedad en el suelo, según explica Burbano (1989). Esto quiere decir que, al haber mayor solubilidad, es mayor la posibilidad de que se pierdan iones de hierro en el riego.
Burbano (1989) menciona que las bajas temperaturas del suelo causan usualmente una disminución en la disponibilidad del microelemento y para este caso ocurre lo contrario: la presencia de cobertura vegetal en un suelo es garantía de aumento en su temperatura, haciendo que aumente la concentración del hierro. Es por esto que en la Figura 13a se observa que el T4 (mulch + EM) tiene la ma- 
yor absorción de hierro con relación a los demás tratamientos.

Los tratamientos que siguen en mayor concentración de hierro son aquellos que tienen fertilizante químico (T6 y T8), y a pesar de que este elemento no está dentro de los componentes principales, sí tiene trazas de hierro, proveniente del URFOS 44. La gallinaza + EM (T5) presenta un nivel tóxico debido a que en los análisis de los abonos aplicados la gallinaza presenta la segunda concentración más alta en hierro. Contrario a lo observado en la Figura 13a, el análisis de varianza múltiple indica que estadísticamente no hay diferencias significativas entre tratamientos, puesto que el valor $\mathrm{F}$ arrojado fue de 0.3755 , lo que permite aceptar que hay una hipótesis nula. Al realizar la prueba de Duncan, evidentemente se detalla que no hay diferencias y que de todas maneras el tratamiento T4 es el que presenta un mayor incremento sobre los demás.

Respecto al Mn, todos tratamientos estén en rangos apropiados según Chapman (1980): esto refleja que en todos los tejidos de la planta existe una adecuada distribución del elemento. El análisis de la variable manganeso para plantas nuevas muestra que estadísticamente no hay diferencias significativas entre los tratamientos, ya que el valor $\mathrm{F}$ encontrado es de 0.3340 , y por lo tanto se acepta la hipótesis nula. Además, de acuerdo a la Figura 13b, no hay gran diferencia entre valores de 0.1 a $0.4 \mathrm{meq} / 10 \mathrm{~g}$, por lo tanto no se ponen las pruebas de Duncan.

\section{Cobre (Cu)}
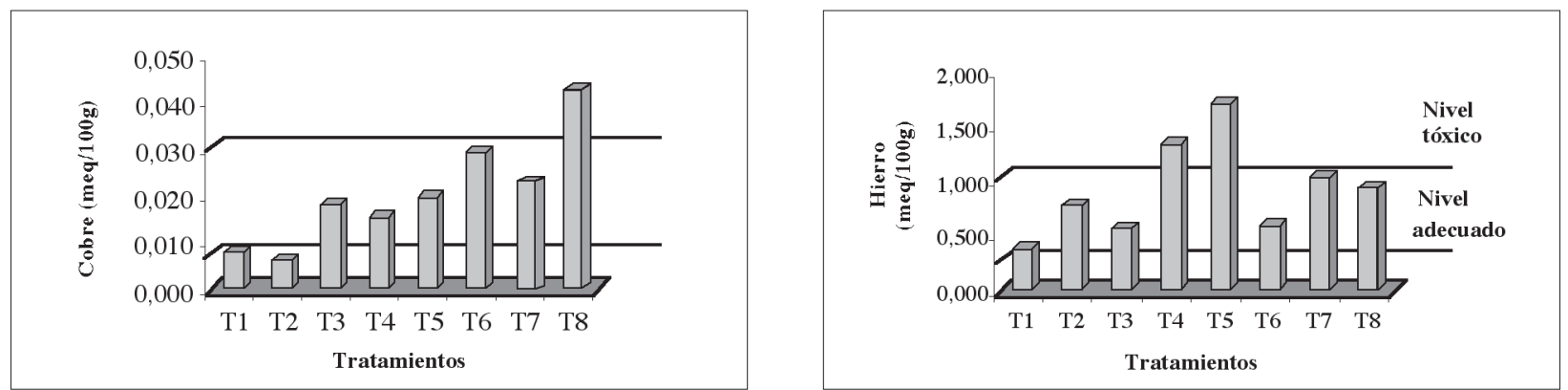

Figura 14. a. Variación de cobre en tejido de plantas nuevas. b. Hierro en plantas antiguas. T1 (testigo), T2 (EM), T3 (compost + EM), T4 (mulch + EM), T5 (gallinaza), T6 (fertilización química), T7 (abonos orgánicos + EM), T8 (abonos orgánicos $+\mathrm{EM}+$ fertilización química).

Chapman (1980) estipula como rango adecuado un valor de 0.008 y $0.031 \mathrm{meq} / 100 \mathrm{~g}$. La Figura $14 \mathrm{a}$ muestra que todos los tratamientos se encuentran dentro del nivel adecuado, exceptuando el tratamiento T2, que se encuentra por debajo de este nivel sin llegar a ser deficiente, y el tratamiento T8, que sobrepasa el nivel adecuado sin ser tóxico.

El análisis de varianza para la evaluación de cobre muestra una probabilidad de 0.1295 , lo que quiere decir que estadísticamente no hay diferencias significativas entre los tratamientos. Por esta razón se rechaza la hipótesis alternativa.

En la Figura 14b se muestra un aumento considerable en la absorción de hierro para los tratamientos T5 (gallinaza + EM) y T4 (mulch + EM). Esto es debido a que en los análisis de los abonos aplicados la gallinaza y el mulch ocupan el segundo y el tercer lugar en abundancia.

En relación con la Figura 13a, correspondiente a la variable hierro para plantas nuevas, se observa que las plantas antiguas absorbieron el metal en menor cantidad en los tratamientos T1, T3, T4, T6, T7 y T8, debido a la fisionomía de las plantas nuevas (mejor estado radical y por lo tanto mayor necesidad de nutrientes), mientras que se presentó una mayor absorción en los tratamientos T2 y T5, donde este último alcanzó un nivel de toxicidad. El análisis de varianza muestra una probabilidad de 0.4397 , indicando que no hay diferencias significativas entre tratamientos; por lo tanto se evidencia la existencia de una hipótesis nula. Los tratamientos 
que presentan mayor incremento en la absorción de manganeso son el T5, debido a que la gallinaza tiene el segundo lugar en contenido de este metal, y el T8, por ser la mezcla de todos los abonos orgánicos, EM y fertilización química.

Se observa que las plantas antiguas que absorbieron menos que las nuevas fueron las de los tratamientos T1, T2, T4, T6 y T8, muy probablemente debido a su fisionomía y su estado radical. El valor arrojado por el análisis de varianza es 0.0191, que permite aceptar la probabilidad de que haya diferencias significativas entre tratamientos que puedan indicar una hipótesis alternativa. La confirmación de esta apreciación se da porque la prueba del rango múltiple de Duncan muestra que efectivamente hay diferencias entre los tratamientos.

\section{Plantas antiguas}

\section{Hierro (Fe) y Manganeso (Mn)}
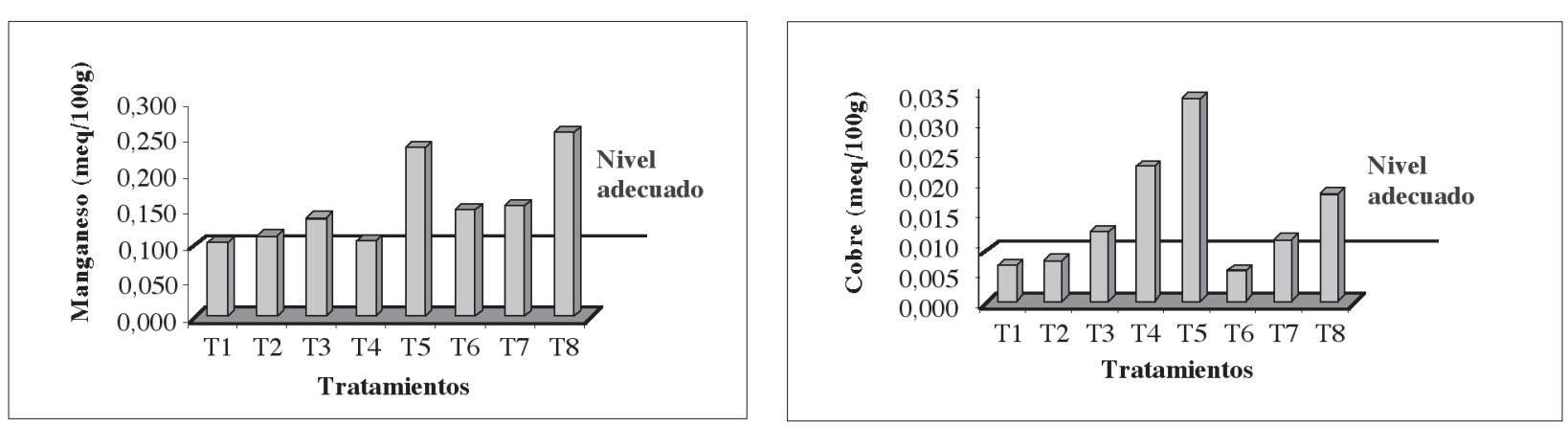

Figura 15. a. Manganeso en tejido de plantas antiguas. b. Variación de cobre en tejido de plantas antiguas. T1 (testigo), T2 (EM), T3 (compost + EM), T4 (mulch + EM), T5 (gallinaza), T6 (fertilización química), T7 (abonos orgánicos + EM), T8 (abonos orgánicos $+\mathrm{EM}+$ fertilización química).

\section{Cobre (Cu)}

En comparación con las plantas nuevas, hubo mayor absorción en los tratamientos T1, T3, T6, T7 y T8, sobresaliendo el T6 Y el T8 debido a la aplicación de fertilización química, mientras que en las plantas antiguas hubo mayor nivel de absorción en los tratamientos T4 y T5, lo que indica un comportamiento inversamente proporcional entre plantas antiguas y nuevas (ver Figuras 14a y 15b). El análisis de varianza muestra una probabilidad $\mathrm{F}$ de 0.1746 , lo que indica que no hay una diferencia estadísticamente significativa entre tratamientos, comprobando así la aceptación de la hipótesis nula.

\section{CONCLUSIONES}

EM (en dosis de 5\% del agua del riego) aporta mejores resultados en el incremento de la capacidad de intercambio catiónico (CIC) en el suelo con la mezcla de los abonos orgánicos (compost, mulch y gallinaza) en dosis de una libra cada uno y con la fertilización química, compuesta por manganeso electrolítico $(0.0002 \mathrm{~g})$, cobre $(0.0002 \mathrm{~g})$, zinc (0.0001g), URFOS 44 (166.66g) y klip-boro (5g). Se encontró además que EM aplicado sólo en la dosis en mención no incrementa la CIC del suelo. Se resalta además que a los dos meses de iniciar la investigación los microorganismos efectivos (EM) aumentaron la capacidad de intercambio catiónico del suelo, en mezcla con los abonos orgánicos (compost, mulch y gallinaza), y este mismo comportamiento se pudo observar al finalizar la investigación (a los cuatro meses).

EM tiende a generar baja de $\mathrm{pH}$ en el suelo cuando es aplicado solo. Sin embargo EM con la mezcla de todos los abonos orgánicos (compost + mulch + gallinaza) aumenta la materia orgánica así como el $\mathrm{pH}$ del suelo. Se encontró además que existe una relación directamente proporcional entre el $\mathrm{pH}$ del suelo y la capacidad de intercambio catiónico en los tratamientos donde se aplicó la mezcla de los materiales orgánicos (compost, mulch y Gallinaza) con EM. 
EM en combinación individual con uno de los abonos orgánicos (compost, mulch o gallinaza) contribuye a una absorción adecuada de calcio en plantas. No obstante, EM aplicado solo o en presencia de la combinación de abonos orgánicos favorece también esta absorción. Se resalta que en plantas nuevas (segundo trasplante) el contenido de calcio se ve favorecido cuando se aplica fertilización química + EM.

EM solo o mezclado con compost no arroja óptimos resultados en la base de magnesio en el suelo. Sin embargo, cuando se aplica gallinaza $+\mathrm{EM}$, tanto en plantas nuevas como antiguas, hay un mayor contenido de magnesio que en los otros abonos.

EM aplicado solo o en mezcla con mulch no presenta niveles de toxicidad en la base de sodio del suelo. No obstante, la fertilización química promueve la sodicidad de las plantas nuevas (trasplantadas dos meses después de iniciada la investigación). Se encontró además que en plantas antiguas (trasplantadas al inicio de la investigación) el aumento de sodio se debió a la aplicación de gallinaza + EM.

Se encontró en todos los tratamientos que el potasio es deficiente, tanto en plantas trasplantadas el mismo día de aplicar los tratamientos (plantas antiguas), como en las trasplantadas después de iniciar la investigación (plantas nuevas); sin embargo, en el suelo hay altos niveles de esta misma base.

El fertilizante químico está influenciando la absorción de las bases en las plantas cuando es aplicado solo con EM. Se encontró además que la mezcla de abonos orgánicos (compost, mulch y gallinaza) + fertilización química + EM aumenta la saturación de bases. Se observó que el fertilizante químico está promoviendo la absorción de magnesio en el suelo, cuando es aplicado con EM.

La gallinaza promueve la absorción de calcio en la relación $\mathrm{Ca} / \mathrm{Mg}$ en el suelo. Y la aplicación de EM solo lleva a una relación próxima a la ideal de $\mathrm{Ca} / \mathrm{K}$ del suelo. Además la gallinaza + EM y la mezcla de los abonos orgánicos (compost, mulch y Gallinaza) + EM promueven más la absorción de potasio en la relación $\mathrm{Ca} / \mathrm{K}$ del suelo. Se encontró que la mezcla de abonos orgánicos + fertilización química + EM promueve más la absorción de calcio en la relación $\mathrm{Ca} / \mathrm{K}$ del suelo.

La aplicación de EM solo mantuvo una relación ideal para evitar desequilibrio en la relación $\mathrm{Mg} / \mathrm{K}$ del suelo. Además mantuvo la relación $\mathrm{Ca}+\mathrm{Mg} / \mathrm{K}$ aproximada al nivel ideal en el suelo.

EM aplicado solo o combinado con compost conservó un nivel adecuado de hierro en plantas (nuevas) después de iniciar la investigación.

EM solo, con cualquiera de los abonos orgánicos o en mezcla con ellos permite que las plantas (nuevas) tengan un nivel adecuado de manganeso.

EM aplicado solo no incrementa la concentración de cobre en plantas (nuevas), mientras que la mezcla de EM con los abonos orgánicos y fertilizantes químicos sobrepasa los niveles adecuados.

EM en presencia de los demás abonos, ya sea con uno solo o en mezcla, incrementa la absorción de hierro y de manganeso en las plantas nuevas. También incrementa la absorción de cobre en las plantas antiguas, mientras que la fertilización química con EM presenta una disminución en estos niveles.

\section{REFERENCIAS BIBLOGRÁFICAS}

Burbano, H. 1989. El suelo: Una visión sobre sus componentes bioorgánicos.. Universidad de Nariño. Pasto.

Calderón, F. 2000. Concepción moderna de la nutrición vegetal. En: Fundamentos para la interpretación de análisis de suelos, plantas y aguas para riego. Bogotá.

Estrada, G. 2000. Fundamentos para la interpretación de análisis de suelos, plantas y aguas para riego. Bogotá.

Fundases. 2006. Fundación de asesoríaspara el sector rural. Disponible en: http://www.fundases.com/ [Consulta enero del 2006]. 
Guerrero, R. 1995. Propiedades químicas de los suelos. Departamento de Suelos y Aguas, Universidad Nacional de Colombia. Bogotá.

Chapman, H. 1980. Diagnostic Criteria For Plant and Soils. Universidad de California. California.

Graetz, H. A. 1996. Manuales para educación agropecuaria: suelos y fertilización. Trillas. México. Pg. 28.

Higa,T. 1994. E. M. Microorganismos eficientes. Disponible en: http://www.fundases.com/p/ em01.html

Instituto Colombiano Agropecuario (ICA). 1979. Suelos y fertilizantes. Bogotá. Pg. 39.

Instituto Colombiano Agropecuario (ICA). 1992. Fertilización en diversos cultivos. Quinta aproximación. ICA. Bogotá.

Instituto Geográfico Agustín Codazzi (IGAC). 1990. Métodos analíticos del laboratorio de suelos. Ministerio de Hacienda y Crédito Público, Instituto Geográfico Agustín Codazzi. Bogotá.

Instituto Geográfico Agustín Codazzi (IGAC). 1990. Métodos analíticos del laboratorio de suelos. Instituto Geográfico Agustín Codazzi. Bogotá. Pp. 1-53, 181.
Instituto Geográfico Agustín Codazzi (IGAC). 1995. Suelos de Colombia. Instituto Geográfico Agustín Codazzi. Bogotá.

León, L. A. 2000. Los elementos mayores nitrógeno, fósforo y potasio en el suelo. En: Fundamentos para la interpretación de análisis de suelos, plantas y aguas para riego. Bogotá. Pg. 193.

Lora. R. 1984. Factores que afectan la disponibilidad de los nutrimentos para las plantas. En: Fertilidad de suelos. Diagnóstico y control. Sociedad Colombiana de la Ciencia del Suelo. Bogotá.

Marín, G. 1990. Fertilidad de suelos con énfasis en Colombia. ICA. Bogotá. Pg, 31.

PROEXANT. 2005. Abonos orgánicos. Disponible en: http://www.proexant.org.ec/Abonos Org\%C3\%A1nicos.html

Soil Survey Staff. 2006. Keys to Soil Taxonomy. USDA-Natural Resources Conservation Service. Washington D. C.

Vázquez, C. \& A. Orozco. 1996. La destrucción de la naturaleza. Fondo de Cultura Económica. México D. F. 


\title{
ANEXOS
}

Anexo A. Análisis de varianza y prueba de comparación de Duncan para la evaluación de cic a la mitad del proceso o 60 días.

\author{
The SAS System \\ General Linear Models Procedure (manova)
}

Dependent Variable: CIC_MIT

$\begin{array}{llll}\text { Source DF } & \text { Sum of Squares } & \text { Mean Square F } & \text { Value oPr }>\text { F } \\ \text { Model } & 7788.09625000 & 112.58517857 & 5.230 .0030^{* *} \\ \text { Error } & 16344.13333333 & 21.50833333 & \\ \text { Corrected Total } & 231132.22958333 & & \end{array}$

The sas System

Duncan's Multiple Range Test for variable: CIC_MIT

Means with the same letter are not significantly different.

$\begin{array}{ccccc}\text { Duncan } & \text { Grouping } & \text { Mean } & \text { N } & \text { TRATAM } \\ & \text { A } & 37.300 & 3 & \text { CMGFq_EM } \\ \text { B } & \text { A } & & & \text { FertQuim_EM } \\ \text { B } & \text { A } & 35.400 & 3 & \text { Gallinaza_EM } \\ \text { B } & \text { C } & 28.833 & 3 & \\ \text { B } & \text { C } & & & \text { Compost_EM } \\ \text { B } & \text { CD } & 27.533 & 3 & \text { CMG_EM } \\ \text { C } & \text { D } & & & \\ \text { C } & \text { D } & 24.100 & 3 & \text { EM } \\ \text { C } & \text { D } & & & \text { Mulch_EM } \\ \text { C } & \text { D } & 24.067 & 3 & \text { CControl } \\ \text { C } & \text { D } & & & 3 \\ \text { C } & \text { D } & 22.933 & 3 & \end{array}$

** Altamente significativo al 1\%. En la prueba de Duncan, promedios de los tratamientos con la misma letra no son significativamente diferentes. * Altamente significativo al 5\%. En la prueba de Duncan, promedios de los tratamientos con la misma letra no son significativamente diferentes. 
Anexo B. Análisis de varianza y prueba de comparación de Duncan para la evaluación de CIC al final del proceso.

$$
\begin{gathered}
\text { The sas System } \\
\text { General Linear Models Procedure (manova) }
\end{gathered}
$$

Dependent Variable: IC FINAL

$\begin{array}{llll}\text { Source DF } & \text { Sum of Squares } & \text { Mean Square } & \text { FValue Pr }>\text { F } \\ \text { Model 7 } & 1004.63958333 & 143.51994048 & 21.330 .0001^{* *} \\ \text { Error } & 16107.68000000 & 6.73000000 & \\ \text { Corrected Total } & 23111 & 2.31958333 & \end{array}$

The sas System

Duncan's Multiple Range Test for variable: CICFINAL

\begin{tabular}{|c|c|c|c|c|}
\hline \multirow[t]{2}{*}{ Duncan } & Grouping & Mean & N & TRATAM \\
\hline & A & 32.067 & 3 & CMGFq_EM \\
\hline & A & & & \\
\hline & A & 29.500 & 3 & FertQuim_EM \\
\hline & A & & & \\
\hline & A & 29.400 & 3 & CMG_EM \\
\hline & B & 23.733 & 3 & Gallinaza_EM \\
\hline & B & & & \\
\hline C & B & 19.733 & 3 & Compost_em \\
\hline$C$ & & & & \\
\hline$C$ & D & 7.533 & & Mulch_EM \\
\hline$C$ & D & & & \\
\hline C & D & 5.667 & 3 & ccontrol \\
\hline & D & & & \\
\hline & D & 14.333 & 3 & EM \\
\hline
\end{tabular}
Means with the same letter are not significantly different.

** Altamente significativo al 1\%. En la prueba de Duncan, promedios de los tratamientos con la misma letra no son significativamente diferentes. * Altamente significativo al 5\%. En la prueba de Duncan, promedios de los tratamientos con la misma letra no son significativamente diferentes. 